\title{
Pricing Double Barrier Parisian Option Using Finite Difference
}

\author{
Xuemei Gao \\ South Western University of Finance and Economics, Chengdu, China \\ Email: gaoxuemei2000@sina.com \\ Received September 15 ${ }^{\text {th }}, 2013$; revised October $15^{\text {th }}, 2013$; accepted October $23^{\text {rd }}, 2013$
}

\begin{abstract}
Copyright (C) 2013 Xuemei Gao. This is an open access article distributed under the Creative Commons Attribution License, which permits unrestricted use, distribution, and reproduction in any medium, provided the original work is properly cited.
\end{abstract}

\begin{abstract}
In this paper, we price the valuation of double barrier Parisian options, under the Black-Scholes framework. The approach is based on fundamental partial differential equations. We reduce the dimension of partial differential equations, then using finite difference scheme to solve the partial differential equations.
\end{abstract}

Keywords: Black-Scholes Model; Double Barrier; Parisian Options; Finite Difference Scheme

\section{Introduction}

It is well known that valuation of financial derivatives, such as options, is one of the major topics in quantitative finance research. A Parisian option is a special kind of barrier options for which the knock-in or knock-out feature is only activated if the underlying price remains continually in breach of the barrier for a pre-specified time period. The valuation of Parisian options can be done by using several different methods: Monte Carlo simulations (Baldi, Caramellino, \& Iovino, 2000), lattices (Avellaneda \& Wu, 1999), Laplace transforms (Zhu \& Chen, 2013) or partial differential equations. An approach based on partial differential equations has been developed by (Wilmott, 1998; Haber, Schönbucher, \& Wilmott, 1999). The options we study in this paper are called double barrier Parisian options. The paper (Chesney, Jeanblanc-Picqué, \& Yor, 1997) introduced the standard Parisian options with two barriers. Double barrier Parisian options are options where the conditions imposed on the assets involve the time spent out of the range defined by two barriers. Double barrier Parisian options have already been priced by (Baldi, Caramellino, \& Iovino, 2000) using Monte Carlo simulations corrected by the means of sharp large deviation estimates, by (Labart \& Lelong, 2009) using Laplace transforms. We use partial differential equations to price double barrier Parisian options. There are two different ways of measuring the time outside the barrier range. One accumulates the time spent in a row and resets the counting whenever the stock price crosses the barrier(s). This type is referred to as continuous double barrier Parisian options. The other adds the time spent in the relevant excursions without resuming the counting from 0 whenever the stock price crosses the barrier(s). These options are named as cumulative double barrier Parisian options. In practice, these two ways of counting time raise different questions about the paths of Brownian motion. In this work, we only focus on continuous knock-out double barrier Parisian call options. We establish the partial differential equation systems for the prices of double barrier Parisian options, and reduce the dimension of partial differential equations, then using finite difference scheme to solve the equations.

\section{The State Space and Boundary Conditions Unavoidable}

The pricing of double barrier Parisian options requires the value of a state variable (clock) $J$, which dictates the time underlying price outside the barrier range (Zhu \& Chen, 2013). When the underlying price $S$ is outside the barrier range, the state variable $J$ starts to accumulate values at the same rate as the passing time $t$, and when the underlying is inside the barrier range, $J$ is reset to zero, and remains zero:

$$
\begin{aligned}
& J=0, \mathrm{~d} J=0, L_{1} \leq S \leq L_{2} \\
& \mathrm{~d} J=\mathrm{d} t, S<L_{1} \text { or } S>L_{2} \\
& 0<L_{1}<L_{2}<\infty
\end{aligned}
$$

where $L_{1}\left(L_{2}\right)$ is a preset lower(up) barrier of the underlying.

According to (Zhu \& Chen, 2013), pricing domain can be defined as:

$$
\begin{aligned}
& I:\{0 \leq S, 0 \leq t \leq T-\bar{J}, J=0\} \\
& I I:\left\{L_{2} \leq S, J \leq t \leq J+T-\bar{J}, 0 \leq J \leq \bar{J}\right\} \\
& I I I:\left\{0 \leq S<L_{1}, J \leq t \leq J+T-\bar{J}, 0 \leq J \leq \bar{J}\right\}
\end{aligned}
$$

$\bar{J}(<\infty)$ is the barrier time triggering parameter. When the variable $J$ reaches $\bar{J}$ the option becomes worthless. $T$ is the expiration time. For simplicity we suppose that $S$ does not jump from $L_{1}$ to $L_{2}$ and does not jump from $L_{2}$ to $L_{1}$. The value of a double barrier Parisian option depends on the underlying price $S$, the current time $t$ and the barrier time $J$, the volatility, risk-free interest rate and the expiry time etc.. Under the Black-Scholes framework, the volatility $\sigma$ is a positive constant, $r$ denotes the risk-free interest rate, the parameter $\delta$ is the dividend rate if the underlying is a stock or the foreign interest rate in case of a currency. $S$ is given by

$$
\mathrm{d} S_{t}=(r-\delta) S_{t} \mathrm{~d} t+\sigma S_{t} \mathrm{~d} W_{t}
$$

where $W_{t}$ is a standard Brownian motion. Let $V_{1}(S, t)$, $V_{2}(S, t, J)$ and $V_{3}(S, t, J)$ denote the option prices in the 
region I, II and III respectively. By applying the Feynman-Kac theorem (Simon, 2000), $V_{1}(S, t)$ should satisfy the classical BS (Black-Scholes) equation

$$
\frac{\partial V_{1}}{\partial t}+\frac{1}{2} \sigma^{2} S^{2} \frac{\partial^{2} V_{1}}{\partial S^{2}}+(r-\delta) S \frac{\partial V_{1}}{\partial S}-r V_{1}=0 .
$$

In region II where the underlying price rises above the barrier $L_{2}$, in region III where the underlying price moves below the barrier $L_{1}$, the barrier time $J$ starts to accumulate. As a result, $V_{2}(S, t, J), V_{3}(S, t, J)$ are governed by a modified Black-Scholes Equation (Haber, Schönbucher \& Wilmott, 1999) respectively,

$$
\frac{\partial V_{i}}{\partial t}+\frac{\partial V_{i}}{\partial J}+\frac{1}{2} \sigma^{2} S^{2} \frac{\partial^{2} V_{i}}{\partial S^{2}}+(r-\delta) S \frac{\partial V_{i}}{\partial S}-r V_{i}=0, i=2,3 .
$$

We show below how the solutions are linked in these three regions. At barrier we impose pathwise continuity of option price, which means the option price does not jump at a barrier. The continuity of the price across the barrier $L_{2}$ demands $\lim _{S \rightarrow L_{2}} V_{1}(S, t)=\lim _{S \rightarrow L_{2}} V_{2}(S, t, 0)$. The continuity of the option price across the barrier $L_{1}$ demands $\lim _{S \rightarrow L_{1}} V_{1}(S, t)=\lim _{S \rightarrow L_{1}} V_{3}(S, t, 0)$.

Appropriate boundary conditions are also needed. In most general form, the option is specified as follows: If the knock out option has not been triggered by expiration $T$, then the option has the price contingent payoff which might also depend on $J$ at expiration; if the knock out option has been triggered during the lifetime of the option, the option pays off the option value at point $(s, t, \bar{J})$. The terminal condition in pricing domain $I$ can be given by the payoff function of a European call of maturity $T$ and exercise price $K$,

$$
V_{1}(S, T)=(S-K)^{+} .
$$

A knock out double barrier Parisian call option is lost if underlying price $S$ made an excursion outside the barrier range older than $\bar{J}$ before $T$,

$$
\begin{aligned}
& \lim _{J \rightarrow \bar{J}} V_{2}(S, t, J)=0, \\
& \lim _{J \rightarrow \bar{J}} V_{3}(S, t, J)=0 .
\end{aligned}
$$

That it would take infinite amount of time for an infinitely large underlying price to fall back to the barrier $L_{2}$, the option must be worth nothing when $S$ becomes very large gives $\lim _{S \rightarrow \infty} V_{2}(S, t, J)=0$. A call option becomes worthless when the underlying price approaches zero, gives $\lim _{S \rightarrow \infty} V_{3}(S, t, J)=0$. The boundary condition at barrier is specified by the so called "reset condition",

$$
\begin{aligned}
& \lim _{S \rightarrow L_{2}} V_{2}(S, t, J)=\lim _{S \rightarrow L_{2}} V_{1}(S, t), \\
& \lim _{S \rightarrow L_{1}} V_{3}(S, t, J)=\lim _{S \rightarrow L_{1}} V_{1}(S, t) .
\end{aligned}
$$

\section{PDE Systems for Pricing Double Barrier Parisian Options}

Under the Black-Scholes framework, the PDE (partial differential equation) systems for the prices of double barrier Parisian options with above boundary conditions have already been established in (Haber, Schönbucher, \& Wilmott, 1999):

$$
\begin{aligned}
& \left\{\begin{array}{l}
\frac{\partial V_{1}}{\partial t}+\frac{\sigma^{2} S^{2}}{2} \frac{\partial^{2} V_{1}}{\partial S^{2}}+(r-\delta) S \frac{\partial V_{1}}{\partial S}-r V_{1}=0 \\
V_{1}(S, T-J)=V_{B S}(S, J) \\
\lim _{S \rightarrow L_{1}} V_{1}(S, t)=\lim _{S \rightarrow L_{1}} V_{3}(S, t, 0) \\
\lim _{S \rightarrow L_{2}} V_{1}(S, t)=\lim _{S \rightarrow L_{2}} V_{2}(S, t, 0)
\end{array}\right. \\
& \left\{\begin{array}{l}
\frac{\partial V_{2}}{\partial t}+\frac{\partial V_{2}}{\partial J}+\frac{\sigma^{2} S^{2}}{2} \frac{\partial^{2} V_{2}}{\partial S^{2}}+(r-\delta) S \frac{\partial V_{2}}{\partial S}-r V_{2}=0 \\
V_{2}(S, t, \bar{J})=0 \\
\lim _{S \rightarrow \infty} V_{2}(S, t, J)=0 \\
\lim _{S \rightarrow L_{2}} V_{2}(S, t, J)=\lim _{S \rightarrow L_{2}} V_{1}(S, t)
\end{array}\right. \\
& \left\{\begin{array}{l}
\frac{\partial V_{3}}{\partial t}+\frac{\partial V_{3}}{\partial J}+\frac{\sigma^{2} S^{2}}{2} \frac{\partial^{2} V_{3}}{\partial S^{2}}+(r-\delta) S \frac{\partial V_{3}}{\partial S}-r V_{3}=0 \\
V_{3}(S, t, \bar{J})=0 \\
\lim _{S \rightarrow 0} V_{3}(S, t, J)=0 \\
\lim _{S \rightarrow L_{1}} V_{3}(S, t, J)=\lim _{S \rightarrow L_{1}} V_{1}(S, t)
\end{array}\right.
\end{aligned}
$$

The above PDE systems are in 3-D and can implified to 2-D PDE systems. $V_{1}$ is already in 2-D. We need to deal with the system governing $V_{2}, V_{3}$. To reduce dimensionality of a PDE system usually requires the application of some sorts of transformation techniques, such as the Fourier transform, the Laplace transform, and so on. Without applying any transformation methods, the pricing domain II is a parallelepipedon, and can be decomposed into infinite many cross-sections (which will be referred to as "slides" hereafter), all of which are of $45^{\circ}$ to both of the plane, $t=0$ and $J=0$ (Zhu \& Chen, 2013). In the pricing domain $I I I$, the positions of the regions are reversed. It is clear that the option value $V_{2}, V_{3}$ at any given point $(S, t, J)$ can be uniquely determined as long as enough information along the every slide passing through that static point is known. In other words, the original 3-D problem can be decomposed into a set of 2-D problems defined on each slide, if viewed from a $45^{\circ}$ rotated coordinate system. Mathematically, to obtain the PDE governing $V_{2}, V_{3}$, in the rotated coordinate system. We can use the directional derivative $\sqrt{2} \frac{\partial V_{2}}{\partial l_{2}}$, $\sqrt{2} \frac{\partial V_{3}}{\partial l_{3}}$ which represents the instantaneous rate of change of the function $V_{2}, V_{3}$ at the point $(t, J)$, in the direction of $(\sqrt{2} / 2, \sqrt{2} / 2)$, to replace the sum of the two partial derivatives $\frac{\partial V_{2}}{\partial t}+\frac{\partial V_{2}}{\partial J}, \frac{\partial V_{3}}{\partial t}+\frac{\partial V_{3}}{\partial J}$, respectively. Furthermore, let $l_{2}{ }^{\prime}=l_{2} / \sqrt{2}, l_{3}^{\prime}=l_{3} / \sqrt{2}$. As a result, the governing equation in the new coordinate system can be written as

$$
\begin{aligned}
& \frac{\partial V_{i}}{\partial l_{i}^{\prime}}+\frac{1}{2} \sigma^{2} S^{2} \frac{\partial^{2} V_{i}}{\partial S^{2}}+(r-\delta) S \frac{\partial V_{i}}{\partial S}-r V_{i}=0 \\
& V_{i}=\left(S, l_{i}^{\prime} ; t\right), i=2,3
\end{aligned}
$$

which is the BS equation. In the new coordinate system, $V_{2}\left(S, l_{2}^{\prime} ; t\right)=V_{2}\left(S, t+l_{2}^{\prime}, l_{2}^{\prime}\right), \quad V_{3}\left(S, l_{3}^{\prime} ; t\right)=V_{3}\left(S, t+l_{3}^{\prime}, l_{3}^{\prime}\right), \quad t$ serves as a parameter. The boundary conditions sets for $V_{2}\left(S, l_{2}^{\prime} ; t\right)$ can be extracted from the corresponding boundary 
conditions that $V_{2}(S, t, J)$ needs to satisfy (Zhu \& Chen, 2013):

$$
\begin{aligned}
& \lim _{S \rightarrow \infty} V_{2}\left(S, l_{2}^{\prime} ; t\right)=0 \\
& \lim _{l_{2}^{\prime} \rightarrow J} V_{2}\left(S, l_{2}^{\prime} ; t\right)=0 \\
& \lim _{S \rightarrow L_{2}} V_{2}\left(S, l_{2}^{\prime} ; t\right)=\lim _{S \rightarrow L_{2}} V_{1}\left(S, t+l_{2}^{\prime}\right)
\end{aligned}
$$

The boundary conditions set for $V_{3}\left(S, l_{3}^{\prime} ; t\right)$ can be extracted from the corresponding boundary conditions that $V_{3}(S, t, J)$ needs to satisfy:

$$
\begin{aligned}
& \lim _{S \rightarrow 0} V_{3}\left(S, l_{3}^{\prime} ; t\right)=0 \\
& \lim _{l_{3}^{\prime} \rightarrow \bar{J}} V_{3}\left(S, l_{3}^{\prime} ; t\right)=0 \\
& \lim _{S \rightarrow L_{1}} V_{3}\left(S, l_{3}^{\prime} ; t\right)=\lim _{S \rightarrow L_{1}} V_{1}\left(S, t+l_{3}^{\prime}\right)
\end{aligned}
$$

Therefore, the2-D PDE systems that govern the price of double barrier Parisian options can be now summarized as:

$$
\begin{aligned}
& \left\{\begin{array}{l}
\frac{\partial V_{1}}{\partial t}+\frac{1}{2} \sigma^{2} S^{2} \frac{\partial^{2} V_{1}}{\partial S^{2}}+(r-\delta) S \frac{\partial V_{1}}{\partial S}-r V_{1}=0 \\
V_{1}(S, T-\bar{J})=V_{B S}(S, \bar{J}) \\
\lim _{S \rightarrow L_{1}} V_{1}(S, t)=\omega_{1}(t) \\
\lim _{S \rightarrow L_{2}} V_{1}(S, t)=\omega_{2}(t)
\end{array}\right. \\
& \left\{\begin{array}{l}
\frac{\partial V_{2}}{\partial l_{2}^{\prime}}+\frac{1}{2} \sigma^{2} S^{2} \frac{\partial^{2} V_{2}}{\partial S^{2}}+(r-\delta) S \frac{\partial V_{2}}{\partial S}-r V_{2}=0 \\
V_{2}(S, \bar{J} ; t)=0 \\
\lim _{S \rightarrow \infty} V_{2}\left(S, l_{2}^{\prime} ; t\right)=0 \\
\lim _{S \rightarrow L_{2}} V_{2}\left(S, l_{2}^{\prime} ; t\right)=\omega_{2}\left(t+l_{2}^{\prime}\right)
\end{array}\right. \\
& \left\{\begin{array}{l}
\frac{\partial V_{3}}{\partial l_{3}^{\prime}}+\frac{1}{2} \sigma^{2} S^{2} \frac{\partial^{2} V_{3}}{\partial S^{2}}+(r-\delta) S \frac{\partial V_{3}}{\partial S}-r V_{3}=0 \\
V_{3}(S, \bar{J} ; t)=0 \\
\lim _{S \rightarrow 0} V_{3}\left(S, l_{3}^{\prime} ; t\right)=0 \\
\lim _{S \rightarrow L_{1}} V_{3}\left(S, l_{3}^{\prime} ; t\right)=\omega_{1}\left(t+l_{3}^{\prime}\right)
\end{array}\right.
\end{aligned}
$$

for $t \in[0, T-\bar{J}], \quad l_{2}^{\prime} \in[0, \bar{J}], \quad l_{3}^{\prime} \in[0, \bar{J}]$.

\section{Algorithm}

The numerical solution to Equations (1)-(3) is implemented using a finite difference scheme in 2-D. Although explicit finite difference schemes are similar to the binomial numerical method in spirit, they are more general and thus more flexible. The method is time-efficient because it is extremely easy to program, and the programs run very quickly. It is suitable for many types of contracts including most common path-dependent derivatives and is trivially-with one extra line of code- extended to American-style early exercise.

In Equation (1), the price $S$ and $t$ are discretized as $\Delta S$, $\Delta t$, respectively. For stability of the scheme, $\Delta t$ has to be chosen small enough. We denote $V^{i, j}$ by the numerical approximation to the option value at $S=i \Delta S, t=j \Delta t$. We call the discrete barrier $\underline{i}$ (i.e., $\underline{i} \Delta S=L_{1}$ ), $\bar{i} \quad$ (i.e., $\bar{i} \Delta S=L_{2}$ ) and $\bar{j}$ (i.e., $\bar{j} \Delta t=\bar{T}-\bar{J}$ ).

$$
\begin{aligned}
& L^{i, j}=\frac{i^{2} \sigma^{2}}{2}\left(V^{i+1, j}-2 V^{i, j}+V^{i-1, j}\right) \\
& +\frac{i(r-\delta)}{2}\left(V^{i+1, j}-V^{i-1, j}\right)-r V^{i, j} \\
& V^{i, j+1}=V^{i, j}+\Delta t L^{i, j}, \forall \underline{i} \leq i \leq \bar{i} .
\end{aligned}
$$

In Equation (2), the price $S$ and $l_{2}^{\prime}$ are discretized as $\Delta S$, $\Delta l_{2}^{\prime}$ respectively. $\Delta l_{2}^{\prime}$ has to be chosen small enough, parameter $t=0, \Delta t, 2 \Delta t, \cdots, \bar{J} \Delta t$. We denote $V^{h, j}$ by the numerical approximation to the option value at $S=h \Delta S$, $l_{2}^{\prime}=j \Delta l_{2}^{\prime}$. We call the discrete barrier $\bar{h}$ (i.e., $\bar{h} \Delta S=L_{2}$ ), and $\bar{j}$ (i.e., $\bar{j} \Delta l_{2}^{\prime}=\bar{J}$ ).

$$
\begin{gathered}
L^{h, j}=\frac{h^{2} \sigma^{2}}{2}\left(V^{h+1, j}-2 V^{h, j}+V^{h-1, j}\right) \\
+\frac{h(r-\delta)}{2}\left(V^{h+1, j}-V^{h-1, j}\right)-r V^{h, j} \\
V^{h, j+1}=V^{h, j}+\Delta l_{2}^{\prime} L^{h, j}
\end{gathered}
$$

In Equation (3), the price $S$ and $l_{1}^{\prime}$ are discretized as $\Delta S$, $\Delta l_{1}^{\prime}$, respectively. $\Delta l_{1}^{\prime}$ has to be chosen small enough, parameter $t=0, \Delta t, 2 \Delta t, \cdots, \bar{J} \Delta t$. We denote $V^{k, j}$ by the numerical approximation to the option value at $S=k \Delta S$, $l_{1}^{\prime}=j \Delta l_{1}^{\prime}$. We call the discrete barrier $\underline{k}$ (i.e., $\underline{k} \Delta S=L_{2}$ ), and $\bar{j}$ (i.e., $\bar{j} \Delta l_{1}^{\prime}=\bar{J}$,

$$
\begin{gathered}
L^{k, j}=\frac{k^{2} \sigma^{2}}{2}\left(V^{k+1, j}-2 V^{k, j}+V^{k-1, j}\right) \\
+\frac{k(r-\delta)}{2}\left(V^{k+1, j}-V^{k-1, j}\right)-r V^{k, j} \\
V^{k, j+1}=V^{k, j}+\Delta l_{3}^{\prime} L^{k, j} .
\end{gathered}
$$

The price $S$ is discretized using equal steps in $\ln S$ and not in $S$.

We compare the prices of a double barrier Parisian out call with $S_{0}=K=100, L_{1}=90, L_{2}=110, \quad r=0.095, \delta=0$ and $T=1$ obtained with our method and Monte Carlo method with 10000 samples. The programs of finite difference schemes run very quickly. Comparison corrected Monte Carlo and finite difference:

\begin{tabular}{cc}
\hline MC Price & FD Price \\
\hline 0.645 & 0.632 \\
1.142 & 1.121 \\
2.747 & 2.732 \\
2.214 & 2.352 \\
3.065 & 3.031 \\
\hline
\end{tabular}

\section{REFERENCES}

Baldi, P. Caramellino, L., \& Iovino, M. G. (2000). Pricing complex barrier options with general features using sharp large deviation estimates. In Monte Carlo and quasi-Monte Carlo methods in scientific computing (3rd ed., pp. 149-162). Claremont, CA: Springer.

Avellaneda, M., \& Wu, L. (1999). Pricing parisian-style options with a lattice method. International Journal of Theoretical and Applied Finance, 2, 1-16. http://dx.doi.org/10.1142/S0219024999000029

Zhu, S. P., \& Chen, W. T. (2013). Pricing Parisian and Parasian options analytically. Journal of Economic Dynamics and Control, 37, 875896. http://dx.doi.org/10.1016/j.jedc.2012.12.005

Wilmott, P. (1998). Derivatives: The theory and practice of financial 
engineering (University ed). Hoboken, NJ: Wiley.

Haber, R. J., Schönbucher, P. J., \& Wilmott, P. (1999). Pricing Parisian options. The Journal of Derivatives, 6, 71-79.

Chesney, M., Jeanblanc-Picqué, M., \& Yor, M., (1997). Brownian excursions and Parisian barrier options. Advances in Applied Microbiology, 29, 165-184.

Labart, C. \& Lelong, J. (2009). Pricing double barrier Parisian options using laplace transforms. International Journal of Theoretical and Applied Finance, 12.

http://dx.doi.org/10.1142/S0219024909005154

Simon, B. (2000). A Faynman-Kac formula for unbounded semigroups. Canadian Mathematical Society Conference Proceedings, 40, 317321. 